IMPORTANT NOTE: Please be aware that slight modifications occurring after Proof correction may occur between this version of the manuscript and the version on the Publisher's website

\title{
Development of a cradle-to-grave approach for acetylated acidic sophorolipid biosurfactants
}

Niki Baccile, ${ }^{1 *}$ Florence Babonneau, ${ }^{1}$ Ibrahim M. Banat, ${ }^{2}$ Katarzyna Ciesielska, ${ }^{3}$ Anne-Sophie Cuvier, ${ }^{1}$ Bart Devreese, ${ }^{3}$ Bernd Everaert, ${ }^{4}$ Helen Lydon, ${ }^{2}$ Roger Marchant, ${ }^{2}$ Christopher A. Mitchell, ${ }^{2}$ Sophie Roelants, ${ }^{4,5}$ Lasse Six, ${ }^{6}$ Elke Theeuwes, ${ }^{7}$ Georgios Tsatsos, ${ }^{8}$ Georgia Eleni Tsotsou, ${ }^{8}$ Brecht Vanlerberghe,${ }^{4}$ Inge N. A. Van Bogaert, ${ }^{5}$ Wim Soetaert ${ }^{4,5^{*}}$

1 Sorbonne Universités, UPMC Univ Paris 06, CNRS, Collège de France UMR 7574, Chimie de la Matière Condensée de Paris, UMR 7574, F-75005 Paris, France. Email :

niki.baccile@upmc.fr

2 School of Biomedical Sciences, University of Ulster, Coleraine BT52 1SA, N. Ireland, UK

3 Laboratory for Protein Biochemistry and Biomolecular Engineering, Faculty of science, Ghent University, K.L. Ledeganckstraat 35, B-9000 Gent, Belgium

4 Bio Base Europe Pilot Plant, Rodenhuizekaai 1, 9042 Gent, Belgium

5 Laboratory for Industrial Biotechnolgy and Biocatalysis (InBio.be), Faculty of Bioscience

Engineering, Ghent University, Coupure Links 653, 9000, Gent, Belgium. Email:

wim.soetaert@ugent.be

6 OWS, Dok Noord 5, 9000 Gent.

7 Ecover Co-ordination Center NV, Steenovenstraat 1a, 2390 Malle, Belgium

8 COSMETIC SP, Ioannou Metaxa 56, Karellas, 19400 Koropi, Greece

\begin{abstract}
Microbial production of biosurfactants represents one of the most interesting alternatives to classical petrol-based compounds due to their low toxicity, high biodegradability and biological production processes from renewable bioresources. However, some of the main drawbacks generally encountered are the low productivities and the small number of chemical structures
\end{abstract}


available, which limits widespread application of biosurfactants. Although chemical derivatisation of (microbial) biosurfactants offers opportunities to broaden the panel of available molecules, direct microbial synthesis is still the preferred option and the use of engineered strains is becoming a valid alternative. In this multidisciplinary work we show the entire process of conception, upscaling of fermentation (150 L) and sustainable purification (filtration), application (foaming, solubilization, antibacterial) and life cycle analysis of acetylated acidic sophorolipids, directly produced by the Starmerella bombicola esterase knock out yeast strain, rather than purified using chromatography from the classical, but complex mixture of acidic and lactonic sophorolipids.

\section{Keywords}

Starmerella bombicola; glycolipids; self-assembly; life cycle analysis; antimicrobial; genetic engineering

\section{Introduction}

Increasing environmental concerns and tightening governing laws have led to increased interest in the development and use of biosurfactants as potential alternatives to chemical surfactants by many industries. ${ }^{1,2}$ At present they are commonly considered as being the next generation of industrial surfactants, because they meet most of the requirements needed for a low environmental impact cradle-to-grave design. Biosurfactants are amphiphilic molecules produced by microorganisms (yeasts, bacteria and fungi) from renewable resources like carbohydrates and natural oils as a carbon source and are generally considered environmentally-friendly due to their high biodegradability and low toxicity. They have effectively been used for some time in a wide range of applications such as oil recovery, bioremediation, oil mobilisation, skin care preparations, cleaning product formulations and, emulsifying agents production among many others. ${ }^{3}$ Four major groups are generally considered: lipopeptides and lipoproteins, like surfactin; polymers, like emulsan; particulate, like vesicles and fimbriae; and lipid containing amphiphilic molecules. The latter category can be further divided into various sub-categories according to the nature of the hydrophilic headgroup (glycolipids, phospholipids, fatty acids, etc.). The reader is addressed towards more general reviews on the synthesis, classification, properties and applications of biosurfactants. ${ }^{4,5}$

Glycolipids are among the largest and most interesting and investigated groups of biosurfactants, because of the higher fermentation yields and versatility in applications. A lot of work has been carried out on rhamnolipids, mannosylerythritol lipids and sophorolipids, just to 
cite the most important compounds in the field. Recent reports of antimicrobial and anti-cancer properties, ${ }^{6} \mathrm{pH}$-dependent self-assembly ${ }^{7}$ and, above all, up-scaling, ${ }^{8,9}$ are undoubtedly some of the main reasons behind the success of these compounds both in fundamental and industrial research efforts. ${ }^{10}$ Among these, sophorolipids, produced by the yeast Starmerella bombicola (previously known as Candida bombicola), are probably the most promising family of biobased amphiphiles. Sophorolipids are constituted of a sophorose head group (2-O- $\beta$-Dglucopyranosyl-D-glucopyranose) attached to a (sub)terminal hydroxylated $\mathrm{C}_{18}$ or $\mathrm{C}_{16}$ fatty acid by a glycosidic linkage between the anomeric C-atom of the sugar and the hydroxyl group of the fatty acid. Their added value with respect to all other compounds combine high production yields (averagely $200 \mathrm{~g} / \mathrm{L}$ ), a congeners mixture of limited complexity compared to others and the knowledge of the Starmerella bombicola biosynthetic pathway as well as the S. bombicola genome map. ${ }^{11}$

Sophorolipids are typically produced by fermentation in the presence of a hydrophobic carbon source and are always constituted of a mixture of structurally related molecules with variation in lactonization between the carboxyl end of the fatty acid and the C4" atom of the sophorose group resulting in a lactonic sophorolipid (Figure S1a) or absence of this lactonization resulting in an open acidic sophorolipid (Figure S1b). Other modifications are the presence (Figure S1c) or absence of acetyl groups at C6' and/or C6" atoms of sophorose, degree of fatty acid saturation (saturated, mono-unsaturated or di-unsaturated, Figure S1d,e) fatty acid chain length and $(\omega)$ or ( $\omega-1)$ hydroxylation of the fatty acid (Figure S1f). ${ }^{12}$

Due to this structural variation, sophorolipids show many interesting applications in a wide range of industrial fields. ${ }^{13}$ Since the structural composition is reflected in the physicochemical properties, the industrial sector is particularly interested in specific structural variants. Lactonic sophorolipids have different biological and physicochemical properties as compared to the acidic form. ${ }^{14}$ In general, lactonic sophorolipids have better surface tension lowering and antimicrobial activity, whereas the acidic ones display better foam producing ability and solubility. ${ }^{15}$ However, lactonic sophorolipids are poorly water soluble, compared to acidic sophorolipids, which are fully soluble in water. While it is possible to obtain $100 \%$ acidic sophorolipids from a natural sophorolipid mixture by a simple alkaline hydrolysis step, it is not possible to obtain the (di-)acetylated acidic congener. In fact, the alkaline hydrolysis will systematically remove all acetyl groups. Meanwhile, up to today, it is impossible to efficiently produce $100 \%$ acetylated acidic sophorolipids by a direct fermentation process with $S$. bombicola. 
In this paper, we present a one-step biological process for the production and purification of acetylated acidic sophorolipids (aSL-COOH) using a strain knocked-out in the lactone esterase gene $(\Delta s b / e)$; a gene mediating lactonization being the last step of the sophorolipid biosynthetic pathway (Figure 1). The major part of sophorolipid biosynthesis takes place intracellularly, in the cytoplasmic environment where acidic non-, mono- and diacetylated sophorolipids are formed. These molecules are subject to active transport by a specific ABCtransporter and once in the extracellular environment, the molecules are exposed to the possible action of the secreted lactone esterase enzyme. ${ }^{11,16}$ In order to fully explore the potential of these novel molecules by specific application testing in view of future real-life usage, sufficient material has to be available and, in addition, at a level of purity suitable for the specific application. A dedicated production process suitable for scale up should also be made available. For this reason, we stepped away from the traditional academic solvent mediated extraction processes $^{15}$ and scaled up a product recovery process, which was developed in this project. This approach allowed us to meet the specific requirements for each application field described in this paper. Indeed, the molecules were evaluated in a broad spectrum addressing both basic properties such as aquatic toxicity and physico-chemical behaviour, as well as their possible usefulness in cosmetic applications and as biological agents.

Four very important features characterize this work, compared to existing works on biosurfactants. First of all, we use a fairly pure sophorolipid mixture composed of nearly $88 \%$ acetylated acidic sophorolipids bearing a C18:1 fatty acid chain. Such a high enrichment of acetylated acidic sophorolipids made available at a scale enabling specific and parallel application testing is not only unique in the literature for this type of compound, but for biosurfactants in general. Secondly, the paper presents quite uniquely the full process pipeline in terms of: 1) expression data (proteomics) of biosynthesis conditions; 2) production optimization; 3) scale-up processing; 4) physico-chemical properties (emulsification, solubilizing properties, foaming, self-assembly); 5) aquatic toxicity; 6) antimicrobial and antibiofilm properties. Thirdly, and probably the most important feature in this field, all experiments noted from 1) through 6) have been carried on the same sample batch, distributed to all co-authors of this work simultaneously. Last but not least a life cycle analysis (LCA) was performed for a hand-washing application, which allowed the determination of hot spots and comparison to reference products on the market. This cradle-to-grave approach using the same compound batch is highly innovative in the field of biosurfactants, where discrepancy among results in the literature is often attributable to natural variations in the sample compositions used for the 
experiments, often combined with poor characterisation, making it difficult to draw reliable conclusions.

\section{Material and methods}

This section is divided into four separate parts, sophorolipid synthesis, its physico-chemical characterization, its biological properties and the prerequisites of LCA analysis. Additional material (analysis of sophorolipid mixtures, protein extraction for multi reaction monitoring, LCMRM analysis, small angle X-ray scattering and transmission electron microscopy under cryogenic conditions) are given in the Supporting Information.

Synthesis of acetylated acidic sophorolipids, batch reference number T21, aSL-COOH (T21).

\section{Strain, growth media and fermentation conditions}

The Starmerella bombicola lactone esterase knock out strain ( $\Delta$ sble) described by Ciesielska et al. ${ }^{16}$ was used for all experiments described in this manuscript. The seed train consisted of two steps, using the production medium described by Lang et al ${ }^{15}$.. The first seeds (50 mL, 48h, $30^{\circ} \mathrm{C}, 200 \mathrm{rpm}$ ) were inoculated from cryovial stocks and transferred to $500 \mathrm{~mL}$ of the same medium $\left(70 \mathrm{~h}, 30^{\circ} \mathrm{C}, 200 \mathrm{rpm}\right)$ in $2 \mathrm{~L}$ flasks. These were inoculated $(3 \%)$ in the main reactor (150 L, Sartorius DCU-3) containing $60 \mathrm{~L}$ of the production medium based on corn steep liquor (CSL) instead of $\mathrm{YE}: 100 \mathrm{~g} / \mathrm{L}$ glucose. $\mathrm{H}_{2} \mathrm{O}, 5 \mathrm{~g} / \mathrm{L}$ dried $\mathrm{CSL}, 1 \mathrm{~g} / \mathrm{L} \mathrm{K} \mathrm{K}_{2} \mathrm{HPO}_{4}, 4 \mathrm{~g} / \mathrm{L}$ $\left(\mathrm{NH}_{4}\right)_{2} \mathrm{SO}_{4}, 0.5 \mathrm{~g} / \mathrm{L} \mathrm{MgSO} 4.7 \mathrm{H}_{2} \mathrm{O}$ and $12 \mathrm{~g} / \mathrm{L}$ rapeseed oil. Aeration and stirring were set to 20 slpm and $500 \mathrm{rpm}$ respectively. After 24 hours of batch fermentation the continuous addition of rapeseed oil was started (0.8 g/L.h) and regulated if necessary while glucose addition was started when its concentration dropped to $40 \mathrm{~g} / \mathrm{L}$ and subsequently maintained above or equal to $30 \mathrm{~g} / \mathrm{L}$. Temperature was maintained constant at $25^{\circ} \mathrm{C}, \mathrm{pH}$ at 3.5 by automatic $\mathrm{NaOH}$ addition after a spontaneous drop during growth and dissolved oxygen concentration was kept above 20 $\%$ by increasing the pressure.

\section{Sophorolipid recovery and purification}

A $0.65 \mu \mathrm{m}$ tangential flow filter element (Pall Kleenpak KT6PSM651HG) was used for cell removal. Further purification by ultrafiltration was performed on a plate and frame filter (DDS Lab-unit, type 20) with $50 \mathrm{kDa}$ (Alfa Laval GR40PP) and 5 kDa (Alfa Laval GR82PP) PES membranes. Hexane extractions were performed and evaporation was done using a rotavapor 
(Buchi, R210/215). The collected sample was given the batch name T21 and used for all tests in this work. T21 is composed out of $88 \%$ of the acetylated acidic form of sophorolipids bearing a C18:1 fatty acid chain (full composition is given in Table S1) and for this reason it will be referred to throughout this work as aSL-COOH (T21).

\section{Physico-chemical characterization of aSL-COOH (T21)}

\section{Hydrophilic-Lipophilic Balance (HLB) value}

A rough estimate of HLB value can be made from the water solubility and dispersibility characteristics of non-ionic surface-active agents. ${ }^{17}$ Their water solubility / dispersibility can also be used as a guide of their expected application as emulsifier, wetting agent, detergent or solubilizer, based on Griffin's classification. ${ }^{18}$ To get an estimate of aSL-COOH (T21) HLB value, the sample was dissolved in water, at room temperature (RT) and at a concentration of 5 $\% \mathrm{w} / \mathrm{w}$. The equilibrium $\mathrm{pH}$ of aSL-COOH (T21) being below 5 , the $\mathrm{pH}$ was then raised adjusted to 5.5 using a $\mathrm{NaOH}$ solution, and the mixture was shaken. The HLB value was estimated based on water solubility according to Griffin's HLB scale and expected applications were assigned based on Griffin's classification scheme for surface-active agents.

\section{Emulsifying properties}

The aSL-COOH (T21) emulsifying properties were investigated by assaying the ability of aSL-COOH (T21) to emulsify a blend of oil and water. The emulsifier was dissolved in water and then added to a biphasic system of oil with water, so as that the respective final concentrations of all components was $3 \% \mathrm{w} / \mathrm{w}$ emulsifier, $82 \% \mathrm{w} / \mathrm{w}$ water and $15 \% \mathrm{w} / \mathrm{w}$ oil. Oils having a range of required HLB (rHLB) values (caprylic capric triglyceride $(\mathrm{rHLB}=5)$, dimethicone $(\mathrm{rHLB}=$ 5), olive oil $(\mathrm{rHLB}=7)$, mineral oil $(\mathrm{rHLB}=10)$, isopropyl palmitate $(\mathrm{rHLB}=11-12)$, were also used in the investigation. The $\mathrm{pH}$ was fixed to 5.0-6.6. The mixtures were manually mixed vigorously. Two series of experiments were run, either at room temperature, or after heating the oil and the water phase to $82{ }^{\circ} \mathrm{C}$. Control emulsions using a commercial emulsifier of similar HLB value (Hostaphat KL340D (Trilaureth-4 Phosphate, HLB= 13-14), or Hostacerin DGL (PEG-10 Polyglyceryl-2-Laurate, HLB= 14-15)) were prepared in parallel. The emulsion stability was visually assessed, following phase separation in the mixture with time. The general microstructure of the emulsion (coarse or fine emulsion and droplet coalescence with time) was also examined at the optical microscope (DIGIMAX model, CETI, Medline Scientific, Oxon, UK) (40x), to assess emulsion quality. A second series of experiments was performed where 
mixtures were homogenised at RT using a homogeniser (Silverson L5M-A, MA, USA). In this set of experiments, emulsion stability was assessed visually following phase separation in the mixture under standard centrifugation conditions (5000 rpm during $10 \mathrm{~min}$ ). The emulsions were also examined using optical microscopy.

\section{Foaming properties}

Foaming of aSL-COOH (T21) was assessed in aqueous solutions. A $12 \% \mathrm{w} / \mathrm{w}$ aqueous solution of a mixture containing the aSL-COOH (T21) and a second surfactant (Cocamidopropyl Betaine), at a weight ratio of actives of 3 aSL-COOH (T21): 1 Cocamidopropyl Betaine, was prepared. As control, a solution containing $12 \% \mathrm{w} / \mathrm{w}$ of a mixture of a surfactant with excellent foaming properties (sodium lauryl ether sulfate (SLES)) and Cocamidopropyl Betaine, at a 3:1 weight ratio of actives, was used. Additional samples were prepared having the same composition as before, plus sunflower oil at two different final concentrations, $1 \% \mathrm{w} / \mathrm{w}$ and $3 \%$ $\mathrm{w} / \mathrm{w}$, to assess foaming properties in the presence of different concentrations of oils. Each sample was shaken in a comparable way. The tubes were visually observed to assess foaming, comparing foam height of aSL-COOH (T21)-containing mixtures to SLES-containing mixtures, in the presence or absence of oil. Comparative foam height assessments in each tube were carried out immediately after shaking, as well as after $10 \mathrm{~min}$ of rest.

\section{Solubilizing properties}

The aSL-COOH (T21) solubilizing properties were investigated after blending the potential solubilizer $(0.75 \% \mathrm{w} / \mathrm{w}$ based on active) with a fragrance (Vanilla Peach or Chamomille Roman Essential Oil, at $0.25 \% \mathrm{w} / \mathrm{w}$ ) and dissolving the blend in water (99\% w/w) at RT, upon vigorous agitation. Azulene (an oil-soluble dye) was used to facilitate visualization of a separated oil phase, where poor solubilization occurred. As control surfactants with good solubilizing properties, we used Polysorbate 20 (Crillet 1, HLB=16.7), PEG-40 Hydrogenated Castor Oil (Croduret 40, HLB= 13-14) and Polysorbate 60 (Crillet 3, HLB= 15), while as control surfactant with poor solubilizing properties was used Polyglyceryl-3-polyricinolate (Dermofeel $\mathrm{PR}, \mathrm{HLB}=4-5)$. A non-surfactant control was also run in parallel. The $\mathrm{pH}$ was fixed to 5.0-6.0. Fragrance solubilization was assessed visually, following separation of an oil layer, or oil droplets, in the mixture. A second series of experiments was also performed using a weight ratio (based on actives with solubilizing properties) 5:1 of solubilizer:fragrance.

\section{Self-assembly}


Samples were prepared by dissolving a given amount of aSL-COOH (T21) in milliQ-grade water. We explored the following concentrations, $0.5 \mathrm{w} \%, 2.5 \mathrm{w} \%$ and $5.0 \mathrm{w} \%$, both at equilibrium $\mathrm{pH}$ (about 4.8, no adjustment after mixing) and $\mathrm{pH} 2$ (adjusted using few $\mu \mathrm{L}$ of $1 \mathrm{M}$ $\mathrm{HCl}$ ). Samples were freshly prepared before SAXS and cryo-TEM analysis, described in more detail below.

\section{Biological properties of aSL-COOH (T21)}

\section{Aquatic toxicity}

Acute immobilisation studies were carried out with Daphnia magna according the OECD Guideline 202 using DAPHTOX F MAGNA (MicroBioTests Inc). aSL-COOH (T21) was compared with two other samples of sophorolipids, a partial hydrolysate (containing $30 \%$ acidic and $70 \%$ lactonic sophorolipids) and complete hydrolysate (containing only non-acetylated acidic sophorolipids) obtained from Ecover (Malle, Belgium). More information on these samples and their synthesis can be found in ref. 19, as described by Develter et al. ${ }^{21}$ For each sample, the Daphnias were exposed to 5 concentrations ranging from $10 \mathrm{mg} / \mathrm{L}$ up to $1000 \mathrm{mg} / \mathrm{L}$ (1000, 333, 100, 33 and $10 \mathrm{mg} / \mathrm{L}$ ) in standard fresh water (Daphtoxkit $\mathrm{F}^{\mathrm{TM}}$ magna tests obtained from MicroBioTests Inc. Belgium). The $\mathrm{pH}$ of the solutions was measured and set to near neutral. Prior to the test, one vial of Ephippia was hatched in standard fresh water for 3 to 4 days at $20^{\circ} \mathrm{C}$ and 6000 lux illumination. Daphnias not older than $24 \mathrm{~h}$ can be used for the test. Before exposure to the sample solutions, the Daphnias were fed with spirulina powder. The test was carried out in a multiwell plate, whereby each row contained 5 . wells, the first well was filled with standard fresh water and the subsequent 4 wells were filled with $10 \mathrm{~mL}$ of the required sample solution (the test was carried out in quadruplet for each concentration). In total, the multiwell plate had 5 rows for each concentration of the sample. In total for each row, 20 active Daphnias are rinsed in the first well, secondly 5 Daphnias are placed in the four remaining wells of that row. The plates are incubated at $20^{\circ} \mathrm{C}$ in the dark. After 24 hours and $48 \mathrm{~h}$ dead and immobile Daphnias were counted for each concentration, the $\mathrm{pH}$ and oxygen concentrations in the solution was also measured. For each test concentration the $24 \mathrm{~h}-\mathrm{EC} 50$ and $48 \mathrm{~h}-\mathrm{EC} 50$ values were calculated with a $95 \%$ confidence limits.

\section{Measurement of growth rate and biofilm formation}

Bacterial strains used in the experiments were Enterococcus faecalis (ATCC 29212), Pseudomonas aeruginosa (PAO1), and Staphylococcus epidermidis (ATCC 38983). Various 
concentrations of aSL-COOH (T21) up to a maximum of $2 \mathrm{w} \%(20 \mathrm{mg} / \mathrm{mL})$ were loaded into 96well microtitre plates (Nunc, ThermoFisher, UK), stored at $4^{\circ} \mathrm{C}$ and used within 5 days of preparation. Each plate contained 6 wells per SL concentration. Nutrient broth $(5 \mathrm{~mL})$ was inoculated with a single colony then transferred to a shaking incubator overnight (16-18 h). Overnight cultures were adjusted, under sterile conditions, to an optical density (OD) of 0.05 at $600 \mathrm{~nm}$; then diluted further and added to the prepared SL test plates, such that the final concentration of culture per well was 1/100th of the 0.05 OD inoculum in $100 \mu \mathrm{L}$ total volume. Following inoculation, the plates were sealed with Parafilm ${ }^{\circledR}$ and incubated in either a shaking or static incubator at $37^{\circ} \mathrm{C}$. Optical density measurements (600nm; FluoStar Omega spectrophotometer coupled with Omega MARS data analysis software version 2.10 R3, BMG LabTech) were recorded for individual plates at each time point for up to $12 \mathrm{~h}$. Following recording of spectrophotometric data, each plate was washed five times in distilled water and air dried overnight. Crystal violet solution $(0.5 \% \mathrm{w} / \mathrm{v} ; 125 \mu \mathrm{L})$ was added to each well and after 45 min the plates were washed five times in distilled water and air dried overnight. Ethanol (95\% $\mathrm{v} / \mathrm{v} ; 150 \mu \mathrm{L}$ ) was added to each well and the plates were incubated at room temperature for 1530 minutes with gentle shaking on a shaking stand, before recording the OD at $595 \mathrm{~nm}$ of the solubilized crystal violet stain in each well. The above procedure was carried out four times in total for each bacterial strain tested, starting with overnight cultures of separate colonies for each strain.

Where appropriate, the data were blank-corrected relative to the mean OD (OD $600 \mathrm{~nm}$ or OD $595 \mathrm{~nm}$ depending on the parameter being measured) for the corresponding blank control. Statistical analysis was carried out using GraphPad Prism (v6.01, GraphPad Software, Inc.). For the growth and biofilm assays, the baseline (t0) value for each individual well was subtracted at each subsequent time point. The mean ODs for each group were then pooled from the separate experiments $(n=4)$ for statistical analysis. Optical densities were compared between treatment $(5-20 \mathrm{mg} / \mathrm{ml} \mathrm{SL})$ and control (no SL) groups at each time point (KruskalWallis test with post-hoc Dunn's multiple comparison). The minimal effective concentration (MEC) was defined as the lowest SL concentration that showed a significant reduction of OD compared to control conditions at two consecutive time points. Subsequently, ODs at each time point were compared to the baseline reading (Kruskal-Wallis test with post-hoc Dunn's multiple comparison).

\section{Life cycle analysis (LCA)}


The environmental impacts associated with the fermentation and purification process described in this paper, but assumed at a larger scale, were calculated according to the LCA approach as defined in ISO 14040:2006 and ISO 14044:2006. In the LCA, yeast extract was used instead of corn steep liquor (CSL) as nitrogen source, as CSL was associated with sterilization and performance problems and would not be taken further along in the development. This difference does not affect the general conclusions of the study. The goal of the LCA study was to determine environmental hot spots when using the surfactant for hand washing (cradle-to-grave), and to compare its total production impacts (cradle-to-gate) to those of the following established detergents, the LCA of which can be found in ref. 20, 21 and 22: soap (sodium salt of fatty acids), alcohol ethoxylates (AE), esterquat, fatty alcohols, fatty alcohol sulfates (FAS), linear alkylbenzene sulphonate (LAS) and alkyl polyglucosides (APG). The associated functional units are 1 hand wash (using $2.3 \mathrm{~g}$ dry weight detergent and $0.64 \mathrm{~L}$ cold tap water) and $1 \mathrm{~kg}$ surfactant (dry weight), respectively. All material and energetic inputs and outputs were included except for capital goods and transport in the foreground system. Background data was gathered from the Ecoinvent database ${ }^{23}$ as applicable for production in Belgium.

The environmental impacts were calculated using the ILCD midpoint v1.04 method and the ReCiPe endpoint (Europe H/A) v1.10 method. The first expresses impacts in terms of climate change, ozone depletion, human toxicity, particulate matter, ionising radiation, photochemical ozone formation, acidification, eutrophication, ecotoxicity, land use and resource depletion. The second method determines damage to human health, ecosystems and resources based on weighted sums of midpoint impacts. Uncertainty of the modelled data and results were determined by use of pedigree matrices and Monte Carlo simulations (1000 runs per result, confidence interval of 95\%). Results with a coefficient of variation larger than $100 \%$ were considered to be highly uncertain and were omitted from the results in order to avoid drawing false conclusions.

\section{Results}

\section{Strain characterization and sophorolipid production.}

In this work we provide a method for the production of $\mathrm{kg}$-scale of $100 \%$ acidic sophorolipids with a high acetylation degree using the Starmerella bombicola lactone esterase knock-out strain ( $\Delta$ sble) described by Ciesielska et al. ${ }^{16}$ as depicted in Figure 1. 
a)

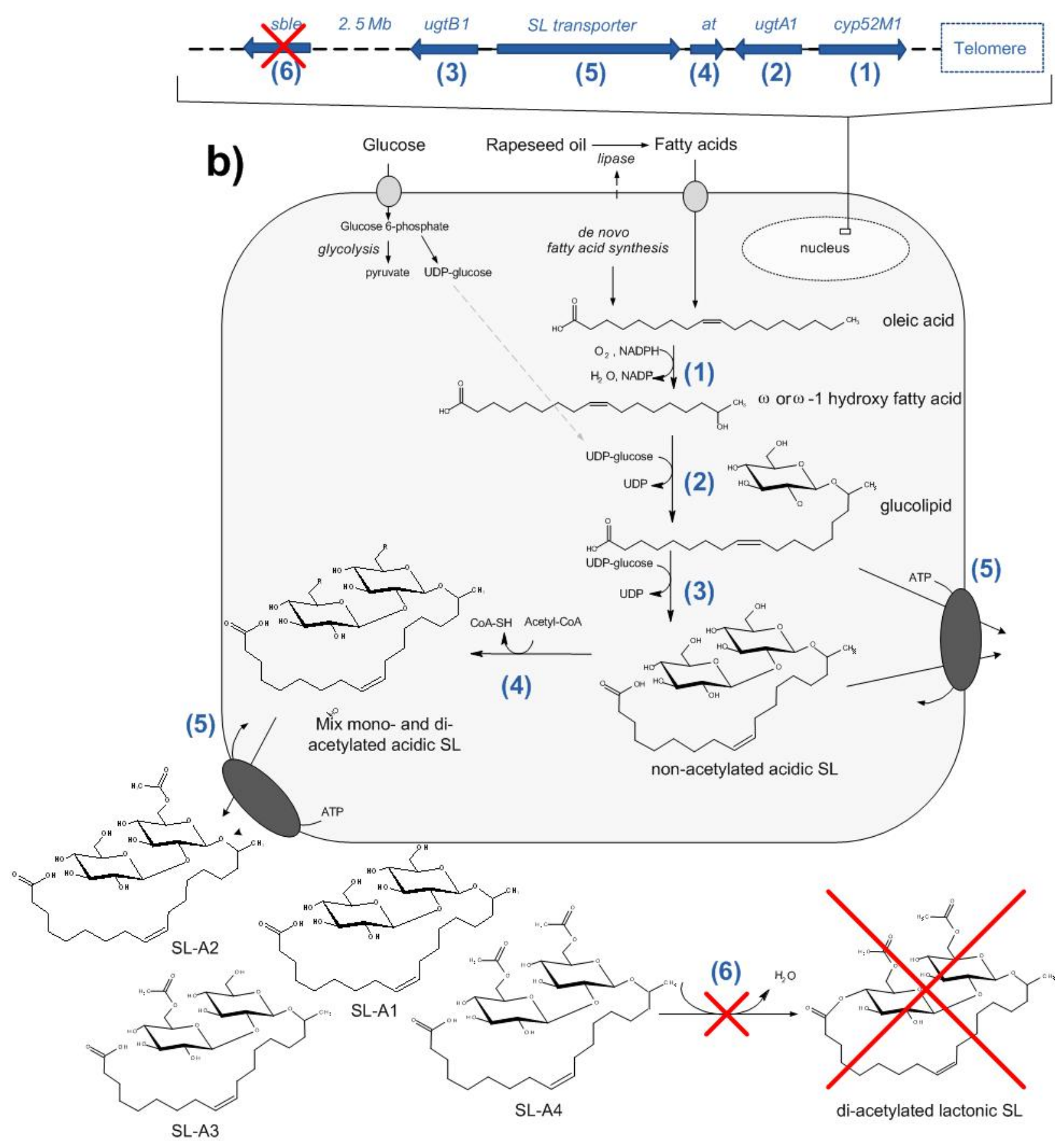

Figure 1 - Overview of the strain engineering strategy to obtain 100\% acetylated acidic SL: (a) genetic makeup of the $\Delta$ sble strain; (b) SL biosynthesis for $100 \%$ acidic SLs with a mixed acetylation pattern: non- (SLA1), mono- (SL-A2 and SL-A3) and di- (SL-A4) acetylated acidic SLs (mainly C18:1 species) are produced and secreted into the extracellular space. (1) cytochrome P450 monooxygenase CYP52M1, (2) glucosyltransferase I, (3) glucosyltransferase II, (4) acetyltransferase, (5) SL transporter, (6) lactone esterase. 
Genetic engineering of $S$. bombicola for the production of (new-to-nature) biosurfactants sometimes brings along undesired side effects on the involved pathway or even on related processes due to inhibition or regulation effects resulting in a drop of the productivity. ${ }^{24}$ In order to verify this for the $\Delta$ sble strain, we characterized it using the Multi Reaction Monitoring (MRM) assay which was recently developed as a molecular tool for $S$. bombicola to determine expression levels of the SL cluster proteins under varying conditions and/or in different strains. ${ }^{25}$ In this case the expression levels of the SL cluster proteins of the $\Delta$ sble strain were compared with those of the S. bombicola wild type (WT) strain. In the MRM assay we target the five proteins of the SL biosynthetic gene cluster: ${ }^{11}$ the SL transporter (MDR), the first UDPglucosyltransferase A1 (UGTA1), the second UDP-glucosyltransferase B1 (UGTB1), the acetyltransferase (AT) and the cytochrome P450 (CYP450). It was recently shown that the $\Delta$ sble strain shows similar productivities as the WT strain. ${ }^{14}$ Here, we confirm that the expression level of its SL cluster proteins is similar as to that of the WT. Surprisingly, the UDPglucosyltransferase A1 (UGTA1) is more abundant in the $\Delta$ sble strain (Figure 2), while the other protein levels are not significantly different. We have strong indications that high levels of SL cluster proteins have a direct connection with high levels of SL productivity (unpublished results).

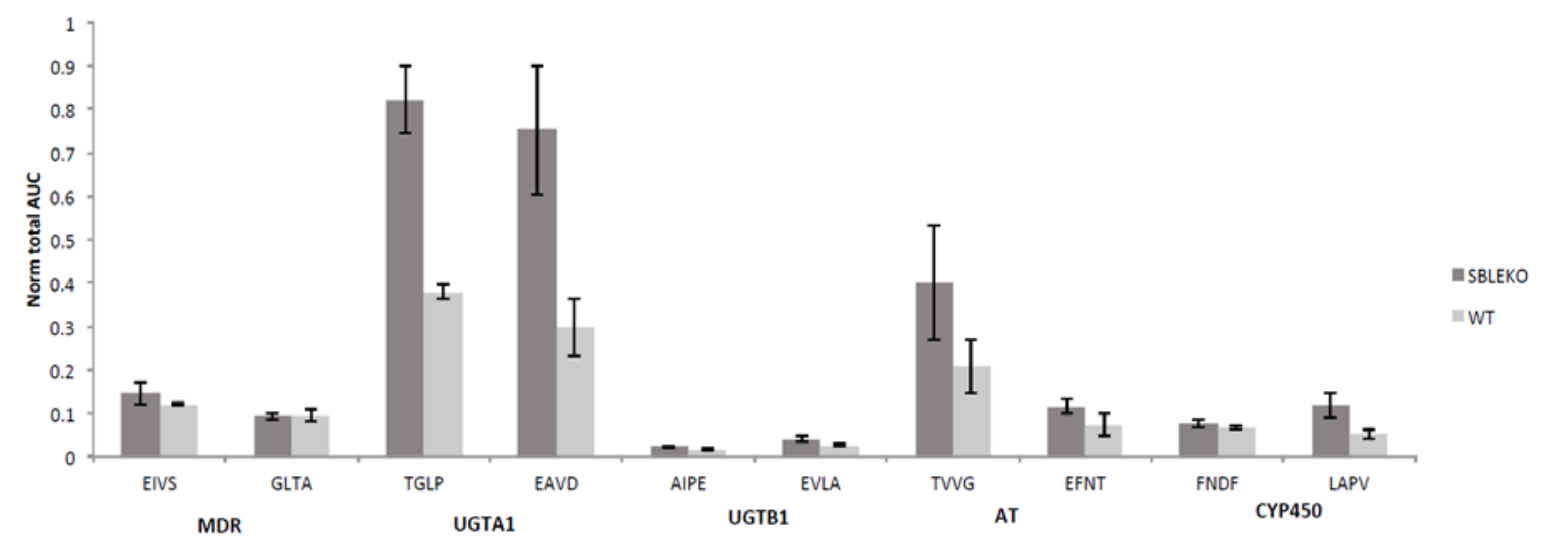

Figure 2 - Relative abundance of SL cluster proteins (MDR-transporter, UGTA1 and UGTB1 glucosyltransferase, AT-acetyltransferase and CYP450- cytochrome P450) in S. bombicola WT and $\Delta$ sble determined by targeted proteomics (LC-MRM). The plot displays the average normalized area-under-thecurve for each peptide used as marker for the different proteins. The error bars plot the SD of all replicate analyses. Target peptides: EIVS: EIVSGSADVGK, GLTL: GLTAASILNEAIDR (MDR); TGLP:TGLPTVEQIK, EAVD:EAVDSIIGNPK (UGTA1); AIPE:AIPEQYDALQTALK, EVLA:EVLATPSYHEK (UGTB1); TVVG:TVVGGVPAR, EFNT:EFNTIASESR (AT); FNDF:FNDFGLGAR, LAPV:LAPVLPLNFR (CYP450).

As mentioned above, thanks to its productivities similar to the WT, this new strain can be used for the large scale production of acetylated acidic sophorolipids, which is not possible 
using the WT, as this will always result in a mixture of acidic and lactonic sophorolipids, although the culture conditions and medium composition can influence the ratio of lactonic/acidic SLs to a certain extent. ${ }^{13,26}$ One could also produce $100 \%$ acidic SLs by applying alkaline hydrolysis on WT-derived SLs in order to open the lactone structure, yet, this will result in acidic SL molecules lacking the acetyl groups, thus generating $100 \%$ non-acetylated acidic SLs (Figure 1: SL-A1). Using this method it is thus not possible to selectively attack the lactone ester function, while leaving the acetyl ester functions intact.

The follow up of important fermentation parameters of the main fermentation $(150 \mathrm{~L}$ scale) are depicted in Figure S2. The run was harvested after $166 \mathrm{~h}$ of fermentation and a sophorolipid concentration of $138 \mathrm{~g} / \mathrm{L}$ was obtained, accounting for a total amount of about 10 $\mathrm{kg}$ of a mixture of acetylated sophorolipids. Due to the high foaming ability of the acetylated acidic SLs, the stirrer and the air flow rate had to be decreased to $400 \mathrm{rpm}$ and $10 \mathrm{slpm}$ respectively after 30 hours of fermentation. To compensate for the resulting lower oxygen transfer rate, the pressure inside the fermenter was increased to 1000 mbar. The oil feed rate was adjusted from $0.8 \mathrm{~g} / \mathrm{L}$.h to $0.25 \mathrm{~g} / \mathrm{L}$.h after $100 \mathrm{~h}$ and stopped after $142 \mathrm{~h}$ of fermentation to allow complete consumption of the oil and free fatty acids towards the end of the fermentation, glucose addition was also stopped after 142 hours.

An overview of the different steps during the recovery and purification of the SLs from the fermentation broth is shown in Figure 3. Filtration was described to be a promising purification process for the SLs produced with the $\Delta$ sble strain at the small scale, ${ }^{14}$ so this process was scaled up to purify the SLs derived from this $150 \mathrm{~L}$ scale fermentation. The cells were removed using filtration (cut off $0.65 \mu \mathrm{m}$ ) and washed to increase the recovery of SLs in the filtrate from $75 \%$ to $95 \%$. Ultrafiltration was subsequently applied on the resulting filtrate to purify the SLs using a two-step filtration process with $50 \mathrm{kDa}$ and $5 \mathrm{kDa}$ cut offs proven to be successful at the small scale. Diafiltration was performed to increase the recovery (50 kDa filtration) and purity (5 kDa filtration) of acetylated acidic sophorolipids. A total recovery of $85 \%$ was obtained using these subsequent filtration steps. A total wash water volume equivalent to $3.5 \times$ the initial cell free broth volume was needed to obtain this value. In an industrial production setting this wash water should be reused. Evaporation was subsequently applied to concentrate the purified SLs. Although one of the aims of this work was to avoid the use of solvents, after this step a necessary hexane extraction step had to be included to remove the remaining oil and free fatty acids (FFAs) from the product, as their presence can influence the product properties. Although oil feeding was stopped one day before the end of the fermentation, these were not completely consumed during the last hours of the fermentation. After this hexane extraction, 
some FFAs still remained associated with the SL product and could not be removed by additional hexane extraction steps, because of strong interaction with the SL product. This shows that it is very important to carefully control the fermentation process in order to avoid the use of solvents and remaining FFAs in the final product, as the latter will have an influence on the properties of the product.

An extra evaporation step was carried out to remove all hexane traces, giving rise to a final concentrated SL product (33\% dry mass). Freeze drying to obtain a dry powdery product was attempted, but was not possible for aSL-COOH (T21), which stays in an oily state (in contrast to non-acetylated SLs or acidic SLs with a lower acetylation degree). The high degree of acetylation thus seems to be responsible for the oily physical state of aSL-COOH (T21). The final composition and purity of the produced T21 batch composed of acetylated acidic SLs can be found in Table S1: $61.1 \%$ and $26.8 \%$ of the product are respectively composed of the diacetylated and mono-acetylated congeners, meaning that about $88 \%$ of the acidic sophorolipids are acetylated and in the C18:1 form. The remaining 12\% is composed of the C18:2 congeners (mono- and di-acetylated, about 9.5\%); the acetylated acidic fully saturated (C18:0) SL only represents $1.2 \%$ while the non-acetylated acidic fraction (C18:1 congener) is as low as $1.5 \%$. The large amount of T21 produced in this work has been dispatched to all partners simultaneously, who independently studied its physico-chemical and biological properties. 


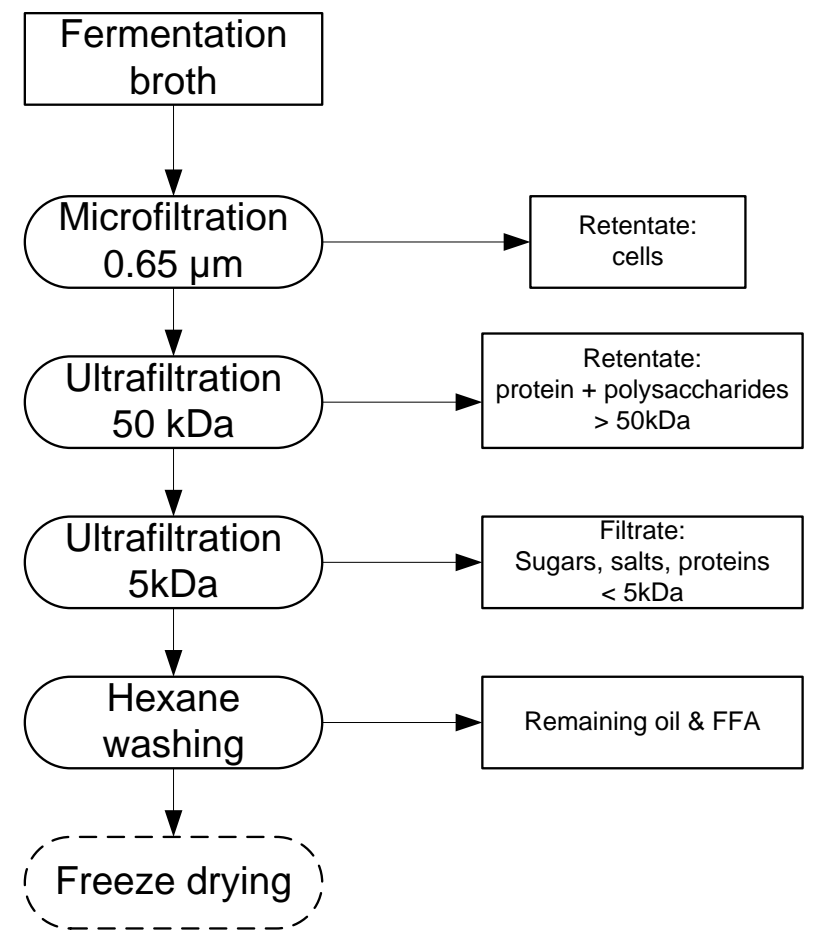

Figure 3 - Schematic representation for the scaled up DSP route to make acetylated acidic sophorolipids produced with the $\Delta$ sble strain.

\section{Physico-chemical properties of aSL-COOH (T21)}

Macroscopic physico-chemical properties have been evaluated for aSL-COOH (T21) in comparison with state-of-the art surface active-agents (see material and method section). Based on its good water solubility, the Hydrophilic-Lipophilic Balance (HLB) value was estimated as higher than 13 (Figure 4A), thus making this compound a potentially interesting oilin-water (O/W) emulsifier, or a solubilizer, according to Griffin's classification. However, direct emulsification tests have shown that O/W emulsions were not stable in the presence of aSL$\mathrm{COOH}$ (T21) under all conditions tested: significant phase separation was observed within few minutes macroscopically, as well as microscopically (droplet coalescence under the optical microscope). Phase separation in a control emulsion using Trilaureth-4 Phosphate as emulsifier, was retarded for at least a month, even at RT. Its microscopic image showed a finer emulsion when compared to the coarse emulsions obtained with aSL-COOH (T21) as emulsifier (Figure 4B). 

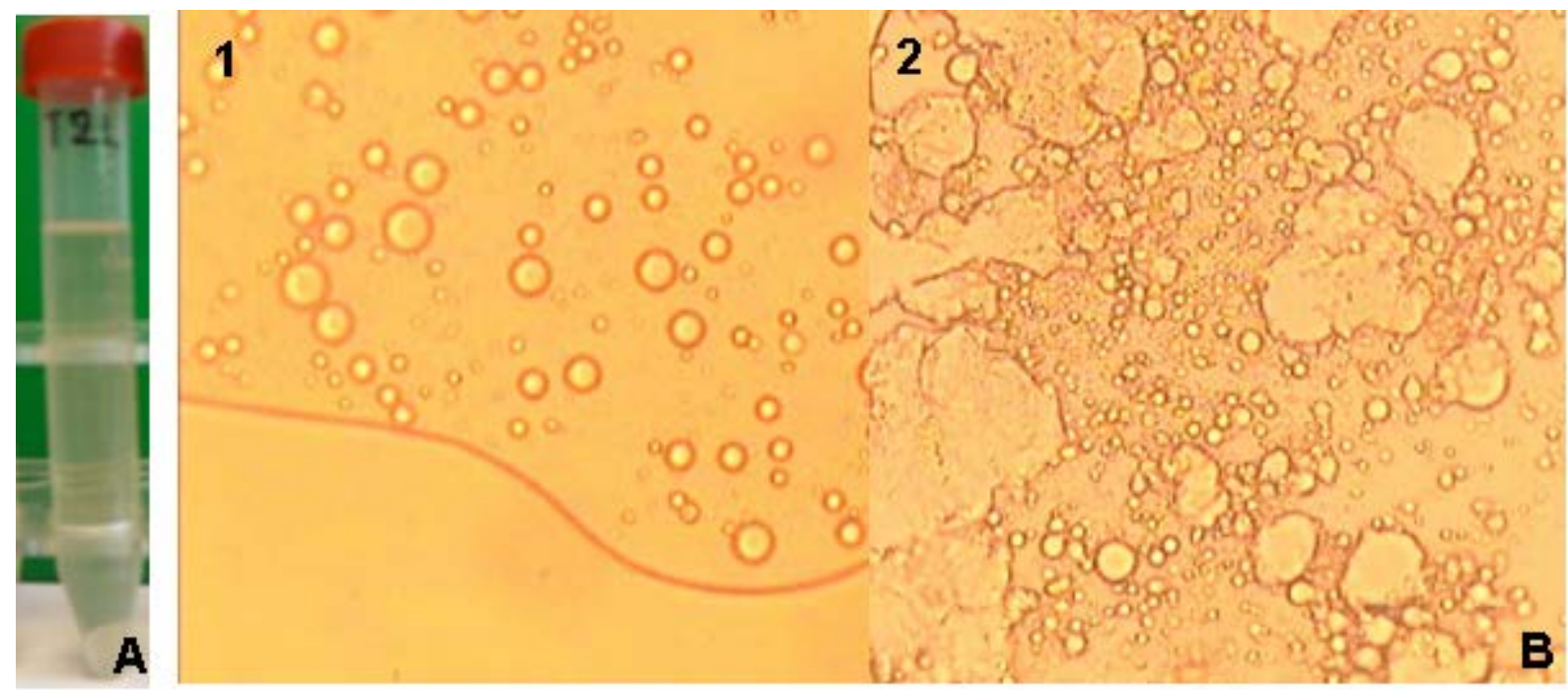

Figure 4 - A) Image of a $5 \%$ w/w aSL-COOH (T21) solution in water, pH 5.5. The aSL-COOH (T21) solution is

clear indicating complete water solubility for aSL-COOH (T21) at the concentration tested. B) Optical microscope images (40x magnification) of (1) mineral oil in water emulsion prepared at $82{ }^{\circ} \mathrm{C}$, where $3 \% \mathrm{w} / \mathrm{w}$ Trilaureth-4 Phosphate was used as emulsifier and (2) mineral oil in water emulsion prepared at $82{ }^{\circ} \mathrm{C}$, where $3 \% \mathrm{w} / \mathrm{w}$ aSL-COOH (T21) (based on actives) was used as emulsifier.

aSL-COOH (T21) foaming performances have also been tested showing a medium foaming ability if compared to SLES (Figure 5). Immediately after shaking, aSL-COOH (T21) foam height was $71 \%$ of the SLES foam height, while after a rest of $10 \mathrm{~min}$ it was $82 \%$ of the fastcollapsing, resting SLES foam height. In the presence of sunflower oil, already at $1.0 \% \mathrm{w} / \mathrm{w}$, aSL-COOH (T21) foam was seriously suppressed, as opposed to SLES foam. Finally, aSL$\mathrm{COOH}$ (T21) solubilization properties towards two fragrances (Vanilla Peach and Chamomille Roman Essential Oil) were tested. Solubilization trials showed that aSL-COOH (T21) has excellent solubilization properties at a 5:1 solubilizer to fragrance ratio. State-of-the art solubilizers demonstrated similar performance at a 3:1 solubilizer to fragrance ratio. 


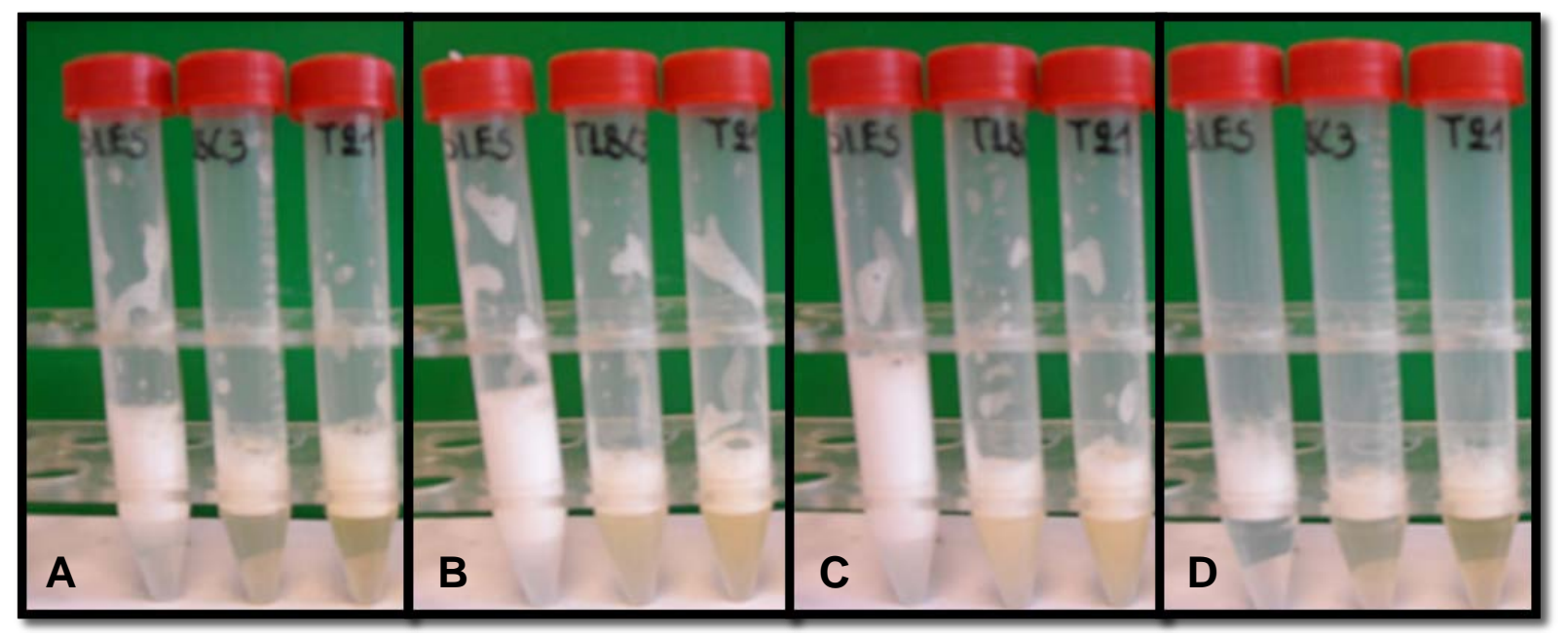

Figure 5 - Images obtained during foaming experiments where the foam height obtained in the presence of SLES and aSL-COOH (T21) was compared without addition of oil ( $A$ and $D$ ), or in the presence of $1 \%$ w/w oil

(B) and $3 \% \mathrm{w} / \mathrm{w}$ oil (C), immediately after vigorous shaking (A, B and C), or after a 10 min rest (D). T18C3 refers to a different, more impure, batch of aSL-COOH, containing non-acetylated acidic sophorolipids and a considerable fraction of hydrophobic impurities as secondary constituents.

The macroscopic properties described above indicate that aSL-COOH (T21) is an interesting hydrophilic compound with a high HLB, thus making it a potential solubilizing agent. To better understand this behaviour, we turn our attention to the study of aSL-COOH (T21) selfaggregation behaviour at a nanoscale level. Considering that an acetylated acidic C18:1 sophorolipid molecule has a very similar structure to the non-acetylated acidic C18:1 sophorolipid, one can make the hypothesis that an ideally pure solution of the former has the same self-assembly behaviour as the latter, that is it forms nanometer-sized micelles. We will study such a hypothesis on the aSL-COOH (T21) compound.

Predictions of the micellar morphology formed by aSL-COOH (T21) can be done using the packing parameter $p,{ }^{27}$ defined as the ratio of $p=\frac{v}{a_{0} \cdot l_{c}}$ with, in a first approximation, $v$ the volume of the hydrocarbon chain, $a_{0}$ the surface area of the hydrophilic headgroup and $I_{c}$ the length of the hydrocarbon chain. The volume of the hydrocarbon chain $v$ can be estimated with $v=13 \mathrm{~V}_{\mathrm{CH} 2}+2 \mathrm{~V}_{\mathrm{CH}}$ (where $\mathrm{V}_{\mathrm{CH} 2}$ is the volume of one $\mathrm{CH}_{2}$ group inside a micelle, $27.5 \AA^{3}$, and $V_{C H}$ is the volume of a $\mathrm{CH}$ group, $22.0 \AA^{3}$ ) to be $v=401.5 \AA^{3}{ }^{28}$ Using the classical Tanford formula, $v=27.4+26.9^{\star} n\left[\AA^{3}\right]$, where $n$ is the number of carbon atoms in the aliphatic chain, one obtains $v=457.8 \AA^{3}$ for a $C_{16}$ chain. Despite the slight disagreement, both values are in the same order of magnitude. The length of a C18:1 aliphatic chain can be estimated to be between $17 \AA$ and $22 \AA$ using the Tanford formula $\left(I_{c}=1.54+1.265^{\star} n\right)$, the difference depending on the 
fact if one considers or not the $120^{\circ}$ bending angle of the C18:1 moiety. Estimation of $a_{0}$ is quite difficult. Cecutti et al. ${ }^{29}$ have estimated the maltoside headgroup area in dodecyl maltoside micelles to be 87 and $50 \AA^{2}$, respectively for the micelle/solvent and at hydrophobic core/sugar headgroup interface. The non-acetylated $\mathrm{C} 18: 1_{\text {cis }}$ sophorose headgroup area was estimated to be $104 \AA^{2}$ at the air-water interface based on SANS data, ${ }^{30}$ while we have measured between 70 and $80 \AA^{2}$ at the dry core/hydrated shell interface of a non-acetylated acidic C18:1 sophorolipid micelle in water. ${ }^{31}$ Since these values do not take into account the presence two acetyl groups, $a_{0}$ is in reality probably slightly larger than $80 \AA^{2}$, probably closer to $100 \AA^{2}$. The calculation of $p$ using average values for $v\left(430 \AA^{3}\right), I_{c}(20 \AA)$ and $a_{0}\left(100 \AA^{2}\right)$ gives $p=0.2$, that is significantly lower than $p<0.3$, the upper limit value for spherical micelles to be stable. ${ }^{27}$ The predicted presence of spherical micelles, demonstrated below, could justify the good solubilization properties measured above.

Small Angle X-ray Scattering (SAXS) can be used to gather information on the selfassembly behaviour of aSL-COOH (T21) as a function of concentration and $\mathrm{pH}$. The data collected at pH 4.8 (obtained after solubilization, with no $\mathrm{pH}$ adjustment) are presented in Figure 6 . The log-log representation of the absolute scale intensity, $I$, versus the scattering vector, $q$, in Figure 6 shows the features of micellar objects in solution, characterized by a typical signal between $0.01<q\left[\AA^{-1}\right]<0.30$ : the micelle/solvent interface is characterized by the $\mathrm{q}^{-4}$ decay at about $0.10 \AA^{-1}$ and the oscillations above $0.10 \AA^{-1}$. The overall I(q) curve also depicts an intense low-q scattering event, most likely due to very large objects in solution simultaneously present with the micelles. These assumptions are confirmed by fitting the SAXS data in the $q>0.01 \AA^{-1}$ region using the form factor of a core-shell ellipsoid of revolution, ${ }^{32}$ previously used to successfully model the self-assembly behaviour of non-acetylated acidic C18:1 sophorolipid micelles. ${ }^{31}$ The hydrophobic core is assumed to be constituted by the fatty acid tail while we assume the hydrophilic shell to be constituted by the acetylated sophorose and possibly the carboxylic acid. One should keep in mind that the presence of the acetyl groups most likely decreases the hydrophilic character of the sophorose headgroup and a core-single shell model is probably an approximation. 

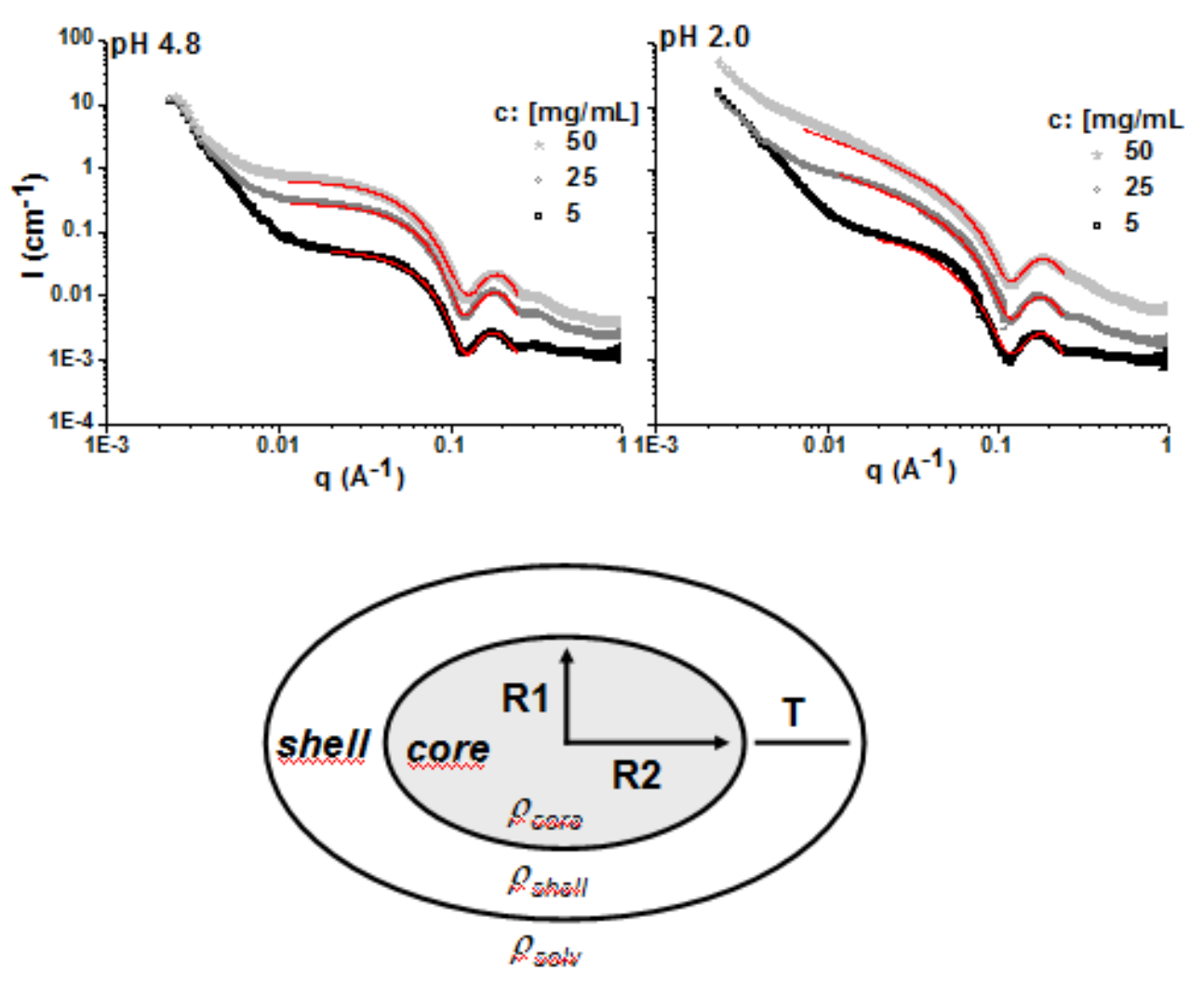

Figure 6 -Log-log representation of I(q) in SAXS experiments performed on the aSL-COOH (T21) sample at three concentrations and at $\mathrm{pH} 4.8$ and $\mathrm{pH}$ 2.0. These curves have been fitted with a core-shell (prolate) ellipsoid of revolution form factor model (solid lines). The results of the fit are given in Table S2. The coreshell ellipsoid model is also sketched: shell and core identify, respectively, the hydrophilic (acetylated sophorose) and hydrophobic (fatty acid) regions of the micelles; R1 and R2 respectively refer to the semiminor and semi-major axes of the core while $\mathrm{T}$ identifies the shell thickness, assumed to be uniform and homogeneous. $\rho$ core, $\rho_{\text {shell }}$ and $\rho_{\text {solv }}$ are the scattering length densities of the core, shell and solvent (water) regions.

Table S2 gives the best fit results for the concentration- and $\mathrm{pH}$-dependent experiments presented in Figure 6. At $\mathrm{pH} 4.8$, the hydrophobic core radius, $\mathrm{R} 1$, is in the range of $11 \AA$ while the hydrophilic shell thickness, T, slightly varies between $15 \AA$ and $18 \AA$. R2 is always slightly bigger than R1, indicating that micelles are effectively prolate ellipsoids. R2 increases with concentration from $21 \AA$ to $40 \AA$, thus indicating that micelles grow slightly with concentration, a common fact and previously found in non-acetylated acidic sophorolipids solutions. ${ }^{31,33}$ Finally, one remarks that at the equilibrium $\mathrm{pH}$, there is no major influence of the concentration on the morphology of the objects (the ellipsoid core-shell model fits well all the curves). Upon decrease of the $\mathrm{pH}$ from 4.8 to 2 , the solutions, transparent at $\mathrm{pH} \mathrm{4.8,} \mathrm{become} \mathrm{slightly} \mathrm{turbid} \mathrm{at} 25$ and 50 
$\mathrm{mg} / \mathrm{mL}$. The cloudiness is typically related to an increase either of the size of the objects in solution and/or of the aggregation.

The typical SAXS response of the system at $\mathrm{pH}=2$ is given in Figure 6 , and which shows a strong increase in the scattering contribution below $q=0.05 \AA^{-1}$, qualitatively indicating an elongation of the micellar aggregates. The fits show that at $\mathrm{pH} 2.0, \mathrm{R} 1$ and $\mathrm{T}$ do not vary significantly, while R2 undergoes a very strong increase. Between 5 and $50 \mathrm{mg} / \mathrm{mL}, \mathrm{R} 2$ increases from $64 \AA$ to more than $2900 \AA$ (while it was between 21 and $40 \AA$ at $\mathrm{pH} 4.8$ ), thus indicating that micelles are actually long cylindrical objects rather than ellipsoids. The fit results at $\mathrm{pH} 2.0$ and $50 \mathrm{mg} / \mathrm{mL}$ should be taken examined with caution; in fact, as one can see in Figure 6 for the corresponding set of data, the fit diverges below $q<0.08 \AA^{-1}$ : it is not possible to fit the entire data set using the core shell ellipsoid form factor, used for all other systems. We believe that, since the slope between $0.02<q\left[\AA^{-1}\right]<0.08$ is nearly -2 , that is characteristics for flat morphologies, rather than cylindrical ones, the system is actually composed of a mixture of micelles and lipid bilayer, possibly vesicles or flat sheets. The heterogeneity of this system is confirmed by the fact that any attempt to model the data using a bilayer form factor was also unsuccessful.

The data in Figure 6 presents another interesting feature. The low-q portion of the spectrum shows a strong scattering contribution and a qualitative analysis of the slope can be very informative on the nature of the scattering objects. Its value can be estimated to be between -3 and -4 , values characteristics of surface fractals, that is an aggregate with a rough surface. ${ }^{34,35}$ This hypothesis will be further discussed in the cryo-TEM section. To conclude on SAXS analysis, pH strongly influences the length of the micelles of aSL-COOH (T21). The SAXS data indicate the presence of micelles coexisting will larger aggregates, which are studied in the following using cryo-TEM experiments.

In order to confirm SAXS data, cryo-TEM experiments have been performed. Figure S3 shows images of typical aggregates that have been found at $50 \mathrm{mg} / \mathrm{mL}$ and $\mathrm{pH} 4.8$. It should be noted that sample observation was not straightforward. First, the amount of sample on the grid was often too important and the thick ice layer on top made it very difficult to record clear-cut images of the self-assembled objects. Second, as illustrated by the white arrow in Figure S3B, organic contamination with, e.g., ethane (used for sample preparation) could easily be mistaken with the sample. Less dense areas were of great interest because individual particles could be distinguished from the large aggregates. Figure S3A may suggest the presence of elongated and/or spherical objects (white circles), the presence of which is also suggested by SAXS. However the exact morphology of these objects is difficult to determine based solely on cryo- 
TEM. The scattering curves in the low-q area had a slope between -3 and -4 , attributed to surface fractals structures. Indeed, the large aggregates inside the white squares in Figure S3 are compatible with the SAXS predictions. Similar aggregates have been observed on nonacetylated acidic sophorolipids at basic $\mathrm{pH} .{ }^{36}$

To conclude on the shapes formed by the acetylated acidic sophorolipids, predictions made by the calculation of the packing parameter $(p<0.3)$ are consistent with the formation of micellar objects, the aspect ratio of which strongly increases with lowering the $\mathrm{pH}$, a fact which was not observed so far on the non-acetylated acidic form of sophorolipids. The introduction of acetyl groups also seem to promote the formation of large aggregated structures, which have been seen before on sophorolipids but under different $\mathrm{pH}$ conditions, that is in the presence of the ionized form of the carboxylic acid end-group. The exact morphology of the structures forming the aggregates is not yet clear, yet.

\section{Biological properties of aSL-COOH (T21)}

Biosurfactants are generally known to be biodegradable and of low toxicity. Meanwhile, they have been employed as antimicrobial agents, and even anti-cancer properties have been described. The acetylated acidic form of sophorolipids can reasonably be presumed poorly toxic, like their non-acetylated counterparts, and the validation of this assumption therefore was very important for their potential large-scale applications. Table S3 shows the results concerning the aquatic toxicity of aSL-COOH (T21), having an EC50 at $24 \mathrm{~h}$ of $580 \mathrm{mg} / \mathrm{L}$ and at $48 \mathrm{~h}$ of 253 $\mathrm{mg} / \mathrm{L}$; these values indicate that aSL-COOH (T21) was practically nontoxic for the aquatic life according to the Hazard Categories related to the aquatic environment. ${ }^{37}$ In terms of comparison, two extra references have been analyzed, a sophorolipid mixture containing $70 \%$ lactonic and $30 \%$ acidic sophorolipids and a non-acetylated acidic sophorolipid sample (the latter contains $1 \%$ of sodium benzoate as a preservative, however non-toxic at this concentration), both obtained from Ecover, and were also found to be non- toxic. Interestingly, early aquatic toxicity tests performed previously on sophorolipids obtained from a commercial source (Soliance, Pomacle, France) and reported by Renkin ${ }^{38}$ were found to have a slight acute toxicity (EC50 after $48 \mathrm{~h}=29,2 \mathrm{mg} / \mathrm{L}$, hazard category: acute 3 ), according to the classification of ref. 37. The Soliance sophorolipids is a mixture of different sophorolipid forms, whereby the acetylated acidic form with a methyl ester function on the fatty acid chain was the most important component, besides the lactonic and deacetylated acidic form. ${ }^{39}$ The reason for this, may be attributable to the impurities present in this particular sample (methyl esters), rather than to an effect related to the compound itself. ${ }^{40}$ 
After testing the aquatic toxicity of aSL-COOH (T21), we have evaluated its antimicrobial and antibiofilm formation properties on three bacterial strains, two Gram-positive, Enterococcus faecalis and Staphylococcus epidermidis, and one Gram-negative, Pseudomonas aeruginosa. For all the strains examined, growth was enhanced in shaking conditions compared to static cultures, whilst biofilm formation was greater in static cultures compared to agitated culture conditions (data not shown): results are therefore shown for the effects of SL treatment in the most optimal conditions for each determination.

Growth and biofilm formation for all bacterial strains were affected by exposure to $<20$ $\mathrm{mg} / \mathrm{mL}$ aSL-COOH (T21) solution known to form micellar aggregates (Figure 6). However, reduction and inhibition of growth and biofilm formation were most effective in Enterococcus faecalis and Staphylococcus epidermidis (Table 1 and Figure 7) with a dose dependent response. High levels of inhibition of growth and biofilm formation were observed at concentrations of $10 \mathrm{mg} / \mathrm{mL}$ and $20 \mathrm{mg} / \mathrm{mL}$. In contrast, growth and biofilm formation were less inhibited in Pseudomonas aeruginosa, although there was still some an observable effect.

Table 1 - The minimal concentrations range of aSL-COOH (T21) which reduced minimal effective concentration (MEC) bacterial growth in agitated cultures (OD600nm) and biofilm quantification in static cultures $\left(O D_{595 \mathrm{~nm}}\right)(\mathrm{n}=4$ per bacterial strain). a For Pseudomonas aeruginosa, a brief inhibition of biofilm formation was observed between 8 and 10 hours of exposure to $0.5 \%$ (5 mg/ml) aSL-COOH (T21) compared to control ( $p<0.05)$, however, no significant inhibition of biofilm formation compared to controls was observed at any time point following exposure to $10-20 \mathrm{mg} / \mathrm{mL}$ aSL-COOH (T21) in this stain.

\begin{tabular}{|c|c|c|}
\hline Strain & Growth $\left(\mathrm{OD}_{600 \mathrm{~nm}}\right)$ MEC & $\begin{array}{c}\text { Biofilm quantification }\left(\mathrm{OD}_{595 \mathrm{~nm}}\right) \\
\text { MEC }\end{array}$ \\
\hline Enterococcus faecalis & $0.5-1 \%$ & $0.5-1 \%$ \\
\hline $\begin{array}{c}\text { Staphylococcus } \\
\text { epidermidis }\end{array}$ & $1-2 \%$ & $0.5-1 \%$ \\
\hline Pseudomonas aeruginosa & $1-2 \%$ & $<1 \%^{\mathrm{a}}$ \\
\hline
\end{tabular}


Growth $\left(O D_{600 \mathrm{~nm}}\right)$
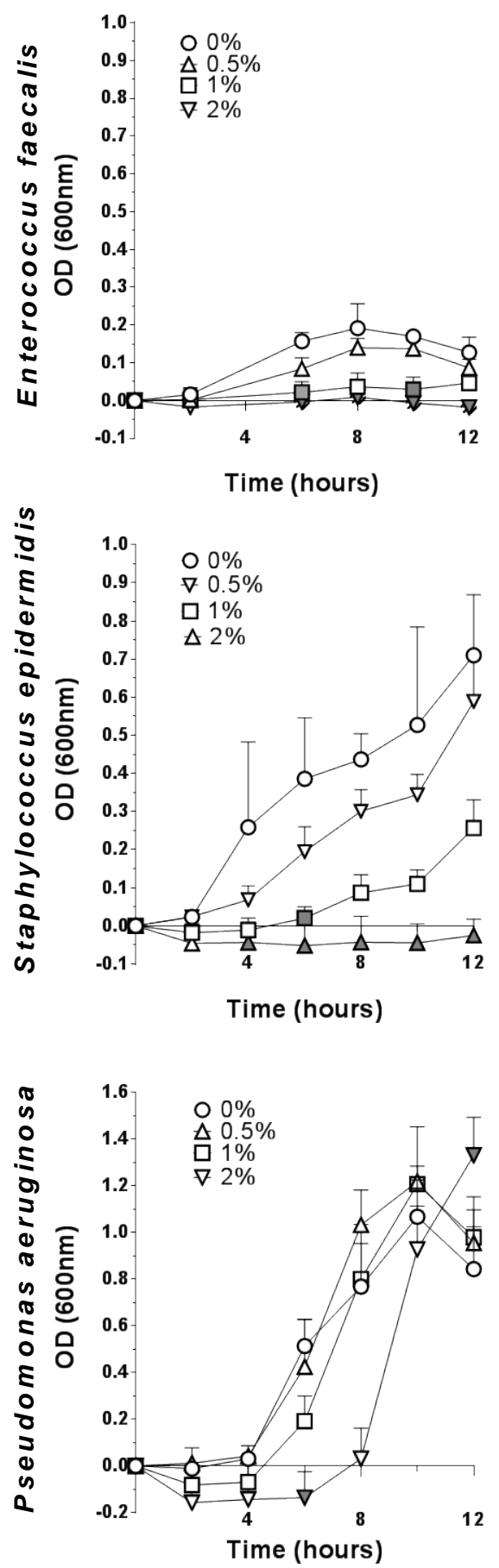

Biofilm formation

(OD $595 \mathrm{~nm}$ )
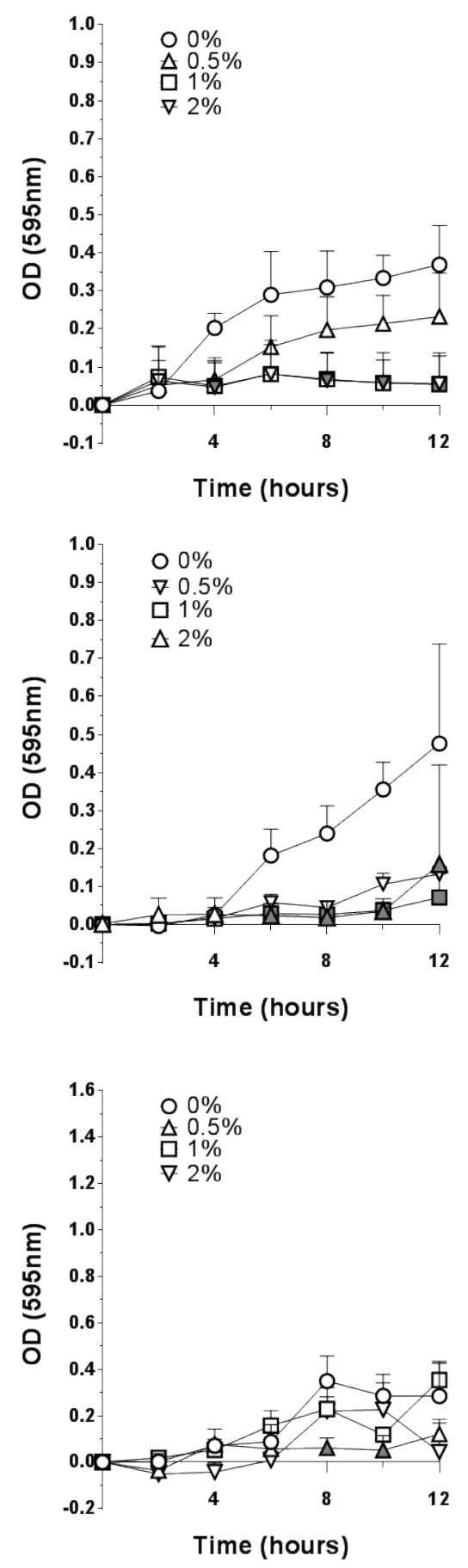

Figure 7 - Growth in agitated cultures $\left(\mathrm{OD}_{600 \mathrm{~nm}}\right.$; left column) and biofilm quantification in static cultures (OD ${ }_{595 n m}$; right column) by Enterococcus faecalis, Staphylococcus epidermidis and Pseudomonas aeruginosa treated with $\leq 2 \%(\leq 20 \mathrm{mg} / \mathrm{mL}$ ) aSL-COOH (T21) (mean \pm standard deviation; $n=4)$. Shaded data points are significantly different to the controls $(p<0.05)$. 
The observation of antibacterial activity in aSL-COOH (T21) is in contrast to a previous report indicating inactivity; ${ }^{41}$ however, we have been able to show that the acidic and lactonic forms of sophorolipid display markedly different, and possibly antagonistic biological activity when used in combination in unpurified preparations (unpublished data). Joshi-Navare \& Prabhune $^{42}$ have reported the inhibitory effects of a mixed sophorolipid preparation on Staphylococcus aureus and Escherichia coli, although their reported MIC values for the sophorolipid alone, at $400 \mu \mathrm{g} / \mathrm{mL}$ and $1 \mathrm{mg} / \mathrm{mL}$ respectively, are lower than the values we report here of $5-20 \mathrm{mg} / \mathrm{mL}$ for MEC values. This disparity may be due to the use of acidic versus mixed lactonic/acidic preparations of sophorolipid since the preparation used by Joshi-Navare \& Prabhane $^{42}$ contained only an estimated $25 \%$ acidic sophorolipid and were very crude preparations with many contaminants, making it impossible to ascribe any effect to the sophorolipids. In addition, it is clear that there was considerable variation in response between different organisms. Treatment with the aSL-COOH (T21) resulted in clear dose-dependent reduction in growth and biofilm formation of Enterococcus faecalis and Staphylococcus epidermidis.

Perhaps the most striking observation is that as great an effect was not apparent for the single Gram negative strain tested, Pseudomonas aeruginosa, in comparison with the two Gram positive strains. This effect is perhaps not so surprising, since $P$. aeruginosa is itself a producer of an active glycolipid biosurfactant, rhamnolipid, which has been implicated in a variety of the physiological activities of the organism including biofilm maintenance and inhibition. ${ }^{43,44}$ In this case, data indicate a delay in growth and/or biofilm formation processes rather than an inhibition, at least at the highest concentration tested. Further experiments should be conducted to determine whether different effects of sophorolipids exist between Gram negative and positive bacteria, with regard to growth and biofilm formation or antibiotic adjuvant activity.

\section{Life Cycle Analysis of Acetylated Acidic Sophorolipids}

The sophorolipids analyzed in the LCA study are a type of biological surfactant, compounds which are used in household detergents as tensides. Although high end or niche applications might be identified in the future, detergency only, one of many potential scenarios, is considered in this study for aSL-COOH.

The hot spots of the cradle-to-grave analysis for hand washing with the sophorolipids produced following the ultrafiltration pathway are shown in Figure 8. The production phase (including sophorolipids and dispenser production) is responsible for most of the impact in every category. The use phase has a minimal impact in all categories, while the end-of-life phase has 
an important impact in some categories, especially climate change and freshwater and marine eutrophication. Strikingly $90 \%$ of the impact from the production phase is caused by the fermentation and even more strikingly it is especially the use of the renewable resources rapeseed oil (47\%) and glucose (41\%) that contributes to this large impact, while only $7 \%$ is derived from electricity. All other input or output streams each contribute less than $5 \%$ of the total impact. The remaining impact for the total production (10\%) is added bit by bit throughout the downstream processing steps, mostly by electricity use.

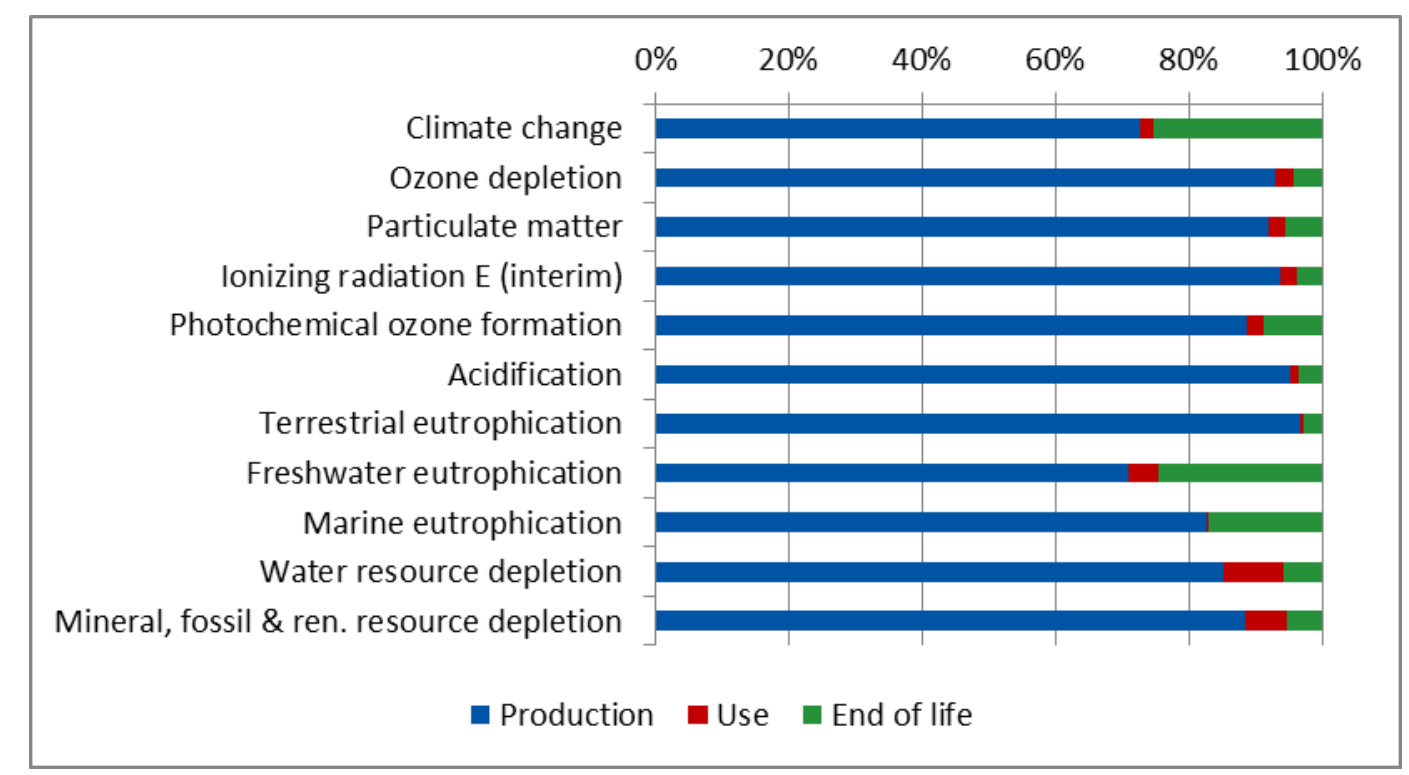

Figure 8 - Cradle-to-grave hot spots for hand-washing application with acidic sophorolipids using fermentation and an ultrafiltration purification pathway.

In a second part of the LCA analysis, the acidic sophorolipids were compared to industrial reference products. Biobased soap is a tenside that has been used for centuries as a household detergent and is therefore considered to be an interesting reference product or benchmark to which sophorolipids can be compared. Other, more recently developed tensides are also included in the comparison: linear alkylbenzene sulfonate (the only completely fossil derived reference product), oleochemicals like ethoxylated alcohol (AE3 and AE7), esterquat, fatty alcohol and fatty alcohol sulfate. Another product to which the acidic sophorolipids are compared, are alkyl polyglucosides (APG). These are so-called first generation biosurfactants, which means that they are produced chemically, but from renewable resources. In contrast to soap, this surfactant is used as a self-emulsifier in cosmetics. An overview of the endpoint results is shown in Figure 9. 


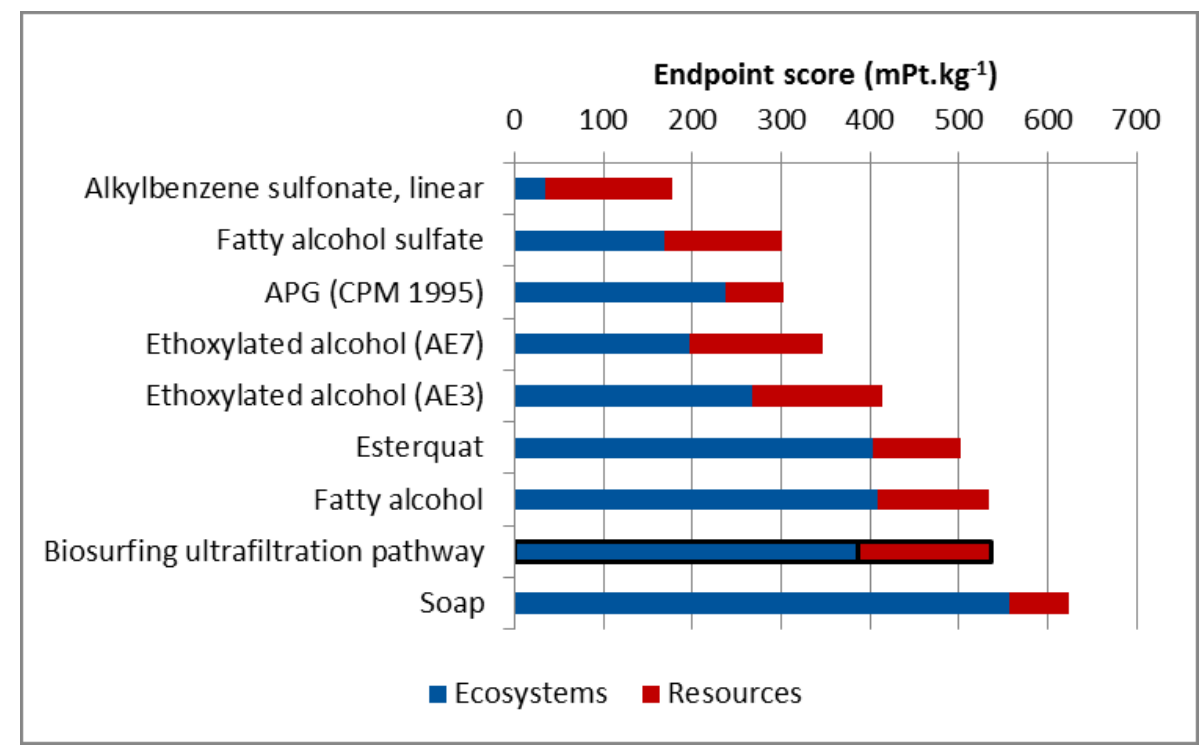

Figure 9 - Endpoint comparison to reference products: overview

At the endpoint level, the acidic sophorolipids perform similar to most of the reference products. In terms of damage to ecosystems, only linear alkylbenzene sulfonate production shows significantly better results, which surprisingly is the only completely fossil derived product in the list. APG, soap and esterquats are produced with less impact to resources. The other products do not differ significantly (overlapping 95\% confidence intervals).

Most of the damage to ecosystems in the sophorolipids production is caused by the production of rapeseed oil and glucose and in particular to the large consumption of water during this phase. In terms of resource use, most of the impact is caused by glucose and electricity production. Another point which is unfavorable to the LCA of sophorolipids is undoubtedly the small volume of produced compound with respect to others, as all data are specific to the mass. In the end, use of second generation raw materials and increased efficiency in the production and purification process will certainly contribute to reduce the hotspots and endpoint scores in the future for this class of materials.

\section{Conclusion}

Glycolipidic biosurfactants obtained by fermentation offer a worthy alternative to petrochemical based surfactants, which are dependent on finite and ever reducing fossil resources. Yet, their overall application is to some extent limited by the lack of structural control and missing availability of specific congeners in sufficient amounts and purity to allow dedicated application tests. In this work we described a scalable (and non-solvent based) method to do so, based on applying a modified strain to obtain $100 \%$ acidic sophorolipids with a high acetylation 
degree and free of lactonic sophorolipids using a Starmerella bombicola lactone esterase knock out strain ( $\Delta$ sble) which produced $138 \mathrm{~g} / \mathrm{L}$ sophorolipids which constitutes of aSL-COOH, C18:1 congener, at about $88 \%$ and no lactonic form of sophorolipid. Aliquots of this compound were used to study its physico-chemical and biological properties.

The aSL-COOH (T21) was found to be non toxic to aquatic environment, yet it can inhibit growth and biofilm formation of both Gram positive and negative bacterial strains. This inhibition was more prominent for the Gram positive bacterial strains. Knowledge of SL-mediated antibacterial action would be of huge benefits in the field of biosurfactant therapeutics as antimicrobial or adjuvant application.

Acidic sophorolipids, aSL-COOH (T21), had very poor O/W emulsion stabilising properties, a medium foaming ability and excellent solubilization properties. We believe that the latter can be explained by the fact that at the equilibrium $\mathrm{pH}$ in water (about 4.8), aSL-COOH (T21) forms micelles, the size and morphology of which vary with $\mathrm{pH}$ : at $\mathrm{pH} 4.8$ the size does not significantly vary with concentration while a more acidic $\mathrm{pH}$ (of 2.0) promotes the formation of large cylindrical objects. However, the SAXS data, combined with cryo-TEM, do not exclude the simultaneous presence of objects with flat morphology.

.Life cycle analysis was carried out to determine the impact of this new production process. The environmental impact was surprisingly rather similar to that of chemical surfactants derived from fossil resources. It was concluded that the use of rapeseed oil and glucose as substrates is the main reason for this impact, with a combined contribution of $78 \%$ of the total endpoint score in terms of damage to ecosystems and damage to resources. The remaining impact was largely caused by electricity use throughout the chain (15\%). Optimization of the conversion efficiency and of the glucose:rapeseed oil ratio holds the most important improvement potential.

This work illustrates the importance and impact of a multidisciplinary approach. As multiple applications were evaluated, all of them influenced the upstream synthesis and purification process in order to obtain the best parameters fitting all needs. The result of this is the development of a cradle to grave process which is green, scalable and applicable for various industries for the synthesis of well characterized biosurfactants with unique properties.

\section{Acknowledgements}

The research leading to these results has received funding from the European Community's Seventh Framework Program (FP7/2007-2013) under Grant Agreement n Biosurfing/289219. SAXS experiments were performed on the SWING beamline at the SOLEIL synchrotron Facility, 
Saint-Aubin, France. Dr. Javier Pérez, local contact at the SWING beamline, is kindly acknowledged for his assistance during the experiments. Patrick Le Griel (Laboratoire de Chimie de la Matière Condensée de Paris, UPMC, Paris, France) is acknowledged for his excellent expertise on the cryo-TEM.

\section{Supporting Information}

Additional information on the materials and method section (HPLC-ELSD, Protein extraction for Multi Reaction Monitoring, MRM, LC-MRM, SAXS, cryo-TEM) are given in the supporting information. Figure S1 shows the typical sophorolipid congeners obtained from the yeast $S$. bombicola, Figure S2 shows the fermentation parameters for the production of acetylated acidic sophorolipids while Figure S3 shows the cryo-TEM images of aSL-COOH (T21). Table S1 (composition of aSL-COOH (T21)), Table S2 (fitting parameters of the SAXS data) and Table S3 (aquatic toxicity tests) are given in the supporting information.

\section{References}

${ }^{1}$ Marchant, R. ; Banat, I. M. Microbial biosurfactants: challenges and opportunities for future exploitation. Trends Biotechnol., 2012, 30, 558-565.

2 Marchant, R.; Banat I. M. Biosurfactants: a sustainable replacement for chemical surfactants? Biotechnol. Lett., 2010, 34, 1597-1605

3 Banat, I. M.; Franzetti, A.; Gandolfi, I.; Bestetti, G.; Martinotti, M. G.; Fracchia, L.; Smyth, T. J.; Marchant, R. Microbial biosurfactants production, applications and future potential. App. Microbiol. Biotechnol., 2010, 87, 427-444

${ }^{4}$ Fracchia, L.; Ceresa, C.; Franzetti, A.; Cavallo, M.; Gandolfi, I.; Van Hamme, J.; Gkorezis, P.; Marchant, R.; Banat, I. M. Industrial Applications of Biosurfactants. "Biosurfactants: Production and UtilizationProcesses, Technologies, and Economics". 2014, In Surfactant Science Series 159, Eds. N. Kosaric and F.V. Sukan. CRC press, Chapter 12, pp 245-260

${ }^{5}$ Marchant, R.; Funston, S.; Uzoigwe, C.; Rahman, P. K. S. M.; Banat, I. M. Production of Biosurfactants from Nonpathogenic Bacteria. "Biosurfactants: Production and Utilization-Processes, Technologies, and Economics", 2014, In Surfactant Science series 159, Eds. N. Kosaric and F.V. Sukan. CRC press, Chapter 5, pp 73-82

${ }^{6}$ Díaz de Rienzo, M.; Stevenson, P.; Marchant, R.; Banat I. M. Antibacterial properties of biosurfactants against selected Gram-positive and -negative bacteria. FEMS Microbiology Letters, 2016, 363, fnv224 
7 Baccile, N.; Selmane, M.; Le Griel, P.; Prévost, S.; Perez, J.; Stevens, C. V.; Delbeke, E.; Zibek, S.; Guenther, M.; Soetaert, W.; Van Bogaert, I. N. A.; Roelants, S. pH-driven self-assembly of acidic microbial glycolipids. Langmuir, 2016, 32, 6343-6359

8 Delbeke, E.; Roelants, S.; Matthijs, N.; Everaert, B.; Soetaert, W.; Coenye, T.; Van Geem, K.; Stevens,, C. Sophorolipid amine oxide production by a combination of fermentation scale-up and chemical modification. Ind. Eng. Chem. Res., 2016, Accepted

9 http://www.sumobrain.com/patents/wipo/Methods-producing-rhamnolipids/WO2015180907A1.html; http://corporate.evonik.de/en/media/search/Pages/news-details.aspx?newsid=59455

${ }^{10}$ Fracchia, L.; Banat, J. J.; Cavallo, M.; Ceresa, C.; Banat, I. M. Potential therapeutic applications of microbial surface-active compounds, AIMS Bioeng., 2015, 2, 144-162

11 Van Bogaert, I. N. A.; Holvoet, K.; Roelants, S. L.; Li, B.; Lin, Y. C.; Van de Peer, Y.; Soetaert, W. The biosynthetic gene cluster for sophorolipids: a biotechnological interesting biosurfactant produced by Starmerella bombicola. Molec. Microbiol., 2013, 88, 501-509

12 Asmer, H. J.; Lang, S.; Wagner, F.; Wray, V. Microbial-production, structure elucidation and bioconversion of sophorose lipids. J. Am. Oil. Chem. Soc., 1988, 65, 1460-1466

${ }^{13}$ Van Bogaert, I. N. A.; Zhang, J.; Soetaert, W. Microbial synthesis of sophorolipids. Process Biochem., 2011, 46, 821-833

14 Roelants, S.; Ciesielska, K.; De Maeseneire, S.; Moens, H.; Everaert, B.; Verweire, S.; Denon, Q.; Vanlerberghe, B.; Van Bogaert, I. N. A.; Van der Meeren, P.; Devreese, B.; Soetaert, W. Towards the industrialisation of new biosurfactants: biotechnological opportunities for the lactone esterase gene from Starmerella bombicola. Biotechnol Bioeng., 2016, 113, 550-559

15 Lang, S.; Brakemeier, A.; Heckmann, R.; Spockner, S.; Rau, U. Production of native and modified sophorose lipids. Chim Oggi-Chem Today, 2000, 18 76-79

${ }^{16}$ Ciesielska, K.; Van Bogaert, I. N. A.; Chevineau, S.; Li, B.; Groeneboer, S.; Soetaert, W.; Van de Peer, Y.; Devreese, B. Exoproteome analysis of Starmerella bombicola results in the discovery of an esterase required for lactonization of sophorolipids. J. Proteom., 2014, 98, 159-174

17 The HLB system- a time saving guide to emulsifier selection. CHEMMUNIQUE (Ed.), 1980, ICI Americas Inc. Delaware, USA

${ }^{18}$ Griffin, W. C. Classification of Surface Active Agents by HLB. J. Soc. Cosmet. Chem., 1949, 1, 311-326

19 Develter, D.; Fleurackers, S. Sophorolipids and rhamnolipids, in "Surfactants form Renewable Resources", 2010, Eds. M. Kjellin M. and I. Johansson, Wiley \& Sons, Ltd.

20 Zah, R.; Hischier, R. Life cycle inventories of detergents, Ecoinvent report no. 12, 2012

${ }^{21}$ Guilbot, J.; Kerverdo, S.; Milius, A.; Escola, R.; Pomrehn, F. Life cycle assessment of surfactants: the case of an alkyl polyglucoside used as a self-emulsifier in cosmetics, Green chem., 2013, 15, p. 33373354.

22 CPM, SPINE LCl dataset: Production of Alkyl Polyglucosides (APG) from coconut oil, 1995

${ }^{23}$ Ecoinvent, Ecoinvent database v3.1, Swiss Centre For Life Cycle Inventories, 2014 
${ }^{24}$ Roelants, S.; De Maeseneire, S.; Ciesielska, K.; Van Bogaert, I. N. A.; Soetaert, W. Biosurfactant gene clusters in eukaryotes: regulation and biotechnological potential. Appl. Microbiol. Biotechnol., 2014, 98, 3449-3461

${ }^{25}$ Roelants, S.; Ciesielska, K.; De Maeseneire, S.; Lodens, S.; Coussement, P.; Pattyn, F.; Saerens, K.; Saey, L.; Verweire, S.; Van Bogaert, I. N. A.; Devreese, B.; Soetaert, W. Development and application of a molecular toolkit for the industrial yeast Starmerella bombicola, Submitted

26 Van Bogaert, I. N. A.; Saerens, K.; De Muynck, C.; Develter, D.; Soetaert, W.; Vandamme, E. J. Microbial production and application of sophorolipids. Appl. Microbiol. Biotechnol., 2007, 76, 23-34.

27 Israelachvili, J. N.; Mitchell, D. J.; Ninham, B. W. Theory of self-assembly of hydrocarbon amphiphiles into micelles and bilayers, Chem. Soc., Faraday Trans. 2, 1976, 72, 1525-1568

${ }^{28}$ Marsh, D. Handbook of Lipid Bilayers, 2013, Second Edition, 448 pp

${ }^{29}$ Cecutti, C.; Focher, B.; Perly, B.; Zemb, T. Glycolipid Self-Assembly: Micellar Structure. Langmuir 1991, 7, 2580-2585

30 Chen, M. ; Dong, C.; Penfold, J.; Thomas, R. K.; Smyth, T. J. P.; Perfumo, A.; Marchant, R.; Banat, I. M.; Stevenson, P.; Parry, A.; Tucker, I.; Campbell, R. A. Adsorption of Sophorolipid Biosurfactants on Their Own and Mixed with Sodium Dodecyl Benzene Sulfonate, at the Air/Water Interface, Langmuir, 2011, 27, 8854-8866

${ }^{31}$ Manet, S.; Cuvier, A.-S.; Valotteau, C.; Fadda, G. C.; Perez, J.; Karakas, E.; Abel, S.; Baccile, N. Structure of Bolaamphiphile Sophorolipid Micelles Characterized with SAXS, SANS, and MD Simulations, J. Phys. Chem. B, 2015, 119, 13113-13133

32 http://www.sasview.org/sasview/user/models/model_functions.html\#coreshellellipsoidxtmodel

33 Baccile, N.; Nassif, N.; Malfatti, L.; Van Bogaert, I. N. A.; Soetaert, W.; Pehau-Arnaudet, G.; Babonneau, F. Sophorolipids: a yeast-derived glycolipid as greener structure directing agents for selfassembled nanomaterials, Green Chem., 2010, 12, 1564-1567

34 Teixeira, J. Small-angle scattering by fractal systems, J. Appl. Crystallogr., 1988, 21, 781-785

35 Gagnepain, J. J.; Roques-Carme, C. Fractal approach to two-dimensional and three-dimensional surface roughness, Wear., 1986, 109, 119-126

${ }^{36}$ Cuvier, A.-S.; Babonneau, F.; Berton, J.; Stevens, C. V.; Fadda, G. C.; Péhau-Arnaudet, G.; Le Griel, P.; Prévost, S.; Perez, J.; Baccile, N. Nanoscale platelet formation by monounsaturated and saturated sophorolipids under basic pH conditions, Chem. Europ. J., 2015, 21, 19265-19277

37 Globally Harmonized System of Classification and Labelling of Chemicals (GHS), Part 4: Environmental hazards, 2015, Sixth revised edition, Copyright @ United Nations

${ }^{38}$ Renkin, M. Environmental profile of sophorolipid and rhamnolipid biosurfactants, La rivista Italiana delle Sostanze Grasse, 2003, 80, 249-252

39 Soliance group (France), personal communication, 24th of October 2011 
${ }^{40}$ Van Bogaert, I. N. A.; Ciesielska, K.; Devreese, B.; Soetaert, W. Sophorolipids Microbial Synthesis and Application, in "Biosurfactants Production and Utilization-Processes, Technologies, and Economics", 2015, Eds. N. Kosaric and F. V. Sukan, CRC Press

${ }^{41}$ Sleiman, J. N.; Kohlhoff, S. A.; Roblin, P. M.; Wallner, S.; Gross, R.; Hammerschlag, M. R.; Zenilman, M. E.; Bluth, M. H. Sophorolipids as antibacterial agents. Annals Clin. Lab. Sci., 2009, 39, 60-63

42 Joshi-Navare, K.; Prabhune, A. A biosurfactant-sophorolipid acts in synergy with antibiotics to enhance their efficiency, BioMed Res. Int., 2013, Article ID 512495, 8 pages, 2013. doi:10.1155/2013/512495

${ }^{43}$ Díaz de Rienzo, M.; Stevenson, P.; Marchant, R.; Banat, I. M. P. aeruginosa biofilm disruption using microbial biosurfactants, J. Appl. Microbiol., 2016, 12, 868-876

44 Díaz de Rienzo, M.; Stevenson, P.; Marchant, R.; Banat, I. M. Effect of biosurfactants on Pseudomonas aeruginosa and Staphylococcus aureus biofilms in a BioFlux channel, Appl. Microbiol. Biotechnol., 2016, $100,5773-5779$ 